九州大学学術情報リポジトリ

Kyushu University Institutional Repository

Identification of novel immune and barrier genes in atopic dermatitis by means of laser capture microdissection

江㟝，仁一

http://hdl. hand le. net/2324/4495983

出版情報：九州大学，2021，博士（医学），論文博士 バージョン:

権利関係: (c) 2014 American Academy of Allergy, Asthma \& Immunology. Published by Mosby, Inc. All rights reserved. 


\title{
Identification of novel immune and barrier genes in atopic dermatitis by means of laser capture microdissection
}

\author{
Hitokazu Esaki, MD, ${ }^{a, b *}$ David A. Ewald, MSc, ${ }^{a, c, d *}$ Benjamin Ungar, BA, ${ }^{a, b}$ Mariya Rozenblit, BA, ${ }^{a, b}$ \\ Xiuzhong Zheng, MSc, ${ }^{a}$ Hui Xu, MSc, ${ }^{a}$ Yeriel D. Estrada, BS, ${ }^{\text {a,b }}$ Xiangyu Peng, MSc, ${ }^{\text {a,b }}$ Hiroshi Mitsui, MD, PhD, ${ }^{a}$ \\ Thomas Litman, PhD, ${ }^{c}$ Mayte Suárez-Fariñas, PhD, ${ }^{a}$ James G. Krueger, MD, PhD, ${ }^{a}$ and \\ Emma Guttman-Yassky, MD, PhD ${ }^{\mathbf{a}, \mathbf{b}} \quad$ New York, NY, and Ballerup and Lyngby, Denmark
}

Background: The molecular signature of atopic dermatitis (AD) lesions is associated with $T_{H} 2$ and $T_{H^{2}} 2$ activation and epidermal alterations. However, the epidermal and dermal AD

transcriptomes and their respective contributions to abnormalities in respective immune and barrier phenotypes are unknown. Objective: We sought to establish the genomic profile of the epidermal and dermal compartments of lesional and nonlesional AD skin compared with normal skin.

Methods: Laser capture microdissection was performed to separate the epidermis and dermis of lesional and nonlesional skin from patients with AD and normal skin from healthy volunteers, followed by gene expression (microarrays and real-time PCR) and immunostaining studies.

Results: Our study identified novel immune and barrier genes, including the IL-34 cytokine and claudins 4 and 8, and showed increased detection of key AD genes usually undetectable on arrays (ie, IL22, thymic stromal lymphopoietin [TSLP], CCL22, and $C C L 26$ ). Overall, the combined epidermal and dermal transcriptomes enlarged the AD transcriptome, adding 674

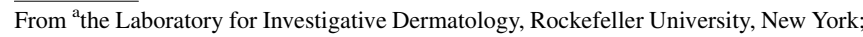
${ }^{b}$ the Department of Dermatology, Icahn School of Medicine at Mount Sinai, New York; ${ }^{c}$ Molecular Biomedicine, LEO Pharma, Ballerup; and ${ }^{d}$ the Center for Microbial Biotechnology, DTU Systems Biology, Technical University of Denmark, Lyngby. *These authors contributed equally to this work.

J.G.K. and M.S.-F. were supported by grant 5UL1RR024143-02 from the National Center for Research Resources (NCRR), a component of the National Institutes of Health (NIH), and the NIH Roadmap for Medical Research. Supported in part by grant no. UL1TR000043 from the National Center for Advancing Translational Sciences (NCATS, NIH Clinical and Translational Science Award [CTSA]) program. E.G.-Y. was supported by the Dermatology Foundation Physician Scientist Career Development Award and by Leo Pharma, and D.A.E. is a joint PhD student of Leo Pharma and DTU and partly funded by the Danish Ministry of Higher Education and Science.

Disclosure of potential conflict of interest: D. A. Ewald is employed by LEO Pharma and has received or has grants pending from the Danish Ministry of Higher Education and Science. T. Litman is an employee of LEO Pharma. J. G. Krueger and his institution have received funding from Novartis, Pfizer, Amgen, Lilly, Merck, Kadmon, Dermira, Boehringer, Innovaderm, Kyowa, BMS, Serono, Janssen, Delenex, AbbVie, Sanofi, Baxter, Paraxel, Xenoport, and Kineta. E. Guttman-Yassky receives compensation for board membership from Sanofi Aventis, Regeneron, Stiefel/GlaxoSmithKline, Medimmune, Celgene, Anacor, and LEO Pharma; receives consultancy fees from Regeneron, Sanofi Aventis, Medimmune, Celgene, Steifel/GlaxoSmithKline, Celsus, BMS, Amgen, and Drais; and has received or has grants pending from Regeneron, Celgene, BMS, and Janssen. The authors declare that they have no other relevant conflicts of interest.

Received for publication September 19, 2014; revised October 28, 2014; accepted for publication October 30, 2014.

Corresponding author: Emma Guttman-Yassky, MD, PhD, Department of Dermatology, Icahn School of Medicine at Mount Sinai Medical Center, 5 E 98th St, New York, NY 10029. E-mail: Emma.Guttman@mountsinai.org.

0091-6749/\$36.00

(C) 2014 American Academy of Allergy, Asthma \& Immunology

http://dx.doi.org/10.1016/j.jaci.2014.10.037
}

upregulated and 405 downregulated differentially expressed genes between lesional and nonlesional skin to the AD transcriptome. We were also able to localize individual transcripts as primarily epidermal (defensin, beta $4 \mathrm{~A}[\mathrm{DEFB} 4 \mathrm{~A}]$ ) or dermal (IL22, cytotoxic T-lymphocyte antigen 4 [CTLA4], and $C C R 7)$ and link their expressions to possible cellular sources. Conclusions: This is the first report that establishes robust epidermal and dermal genomic signatures of lesional and nonlesional AD skin and normal skin compared with whole tissues. These data establish the utility of laser capture microdissection to separate different compartments and cellular subsets in patients with AD, allowing localization of key barrier or immune molecules and enabling detection of gene products usually not detected on arrays. (J Allergy Clin Immunol 2015;135:153-63.)

Key words: Atopic dermatitis, laser capture microdissection, IL-34, claudins 8 and 4, immune, barrier

Atopic dermatitis (AD) is the most common inflammatory skin disease. ${ }^{1,2}$ Although its pathogenesis is not fully understood, both barrier and immune components have been suggested to play key roles in $\mathrm{AD}$, as indicated by the "outside-in" and "inside-out" hypotheses. ${ }^{3-10}$ Whereas barrier-related molecules are largely epidermal, inflammatory responses are derived from both the epidermal (ie, keratinocytes and Langerhans cells [LCs]) and dermal (ie, T cells and dendritic cells [DCs]) compartments.

Using genomic analyses on whole tissue/bulk samples, we have previously shown that the $\mathrm{AD}$ phenotype/transcriptome is associated with polar immune activation of $\mathrm{T}_{\mathrm{H}} 2 / \mathrm{T}_{\mathrm{H}} 22$, as well as $T_{H} 1$ and $T_{H} 17$, pathways and corresponding epidermal alterations (epidermal hyperplasia and abnormal differentiation). ${ }^{11-14}$ However, bulk sample genomic analysis (by using microarrays and real-time PCR [RT-PCR]) presents some limitations. First, it is difficult to determine whether altered gene expression is due to expansion (hypertrophy) of one tissue compartment versus altered gene expression at the cellular level. Second, it cannot localize a particular gene/transcript to an epidermal/dermal compartment. Finally, low-abundance genes are often present at less than the detection level of conventional microarrays because of dilution of mRNA within full-thickness samples with more dominant products.

Laser capture microdissection (LCM) is an established technique for procuring subpopulations of tissues/cells of interest 


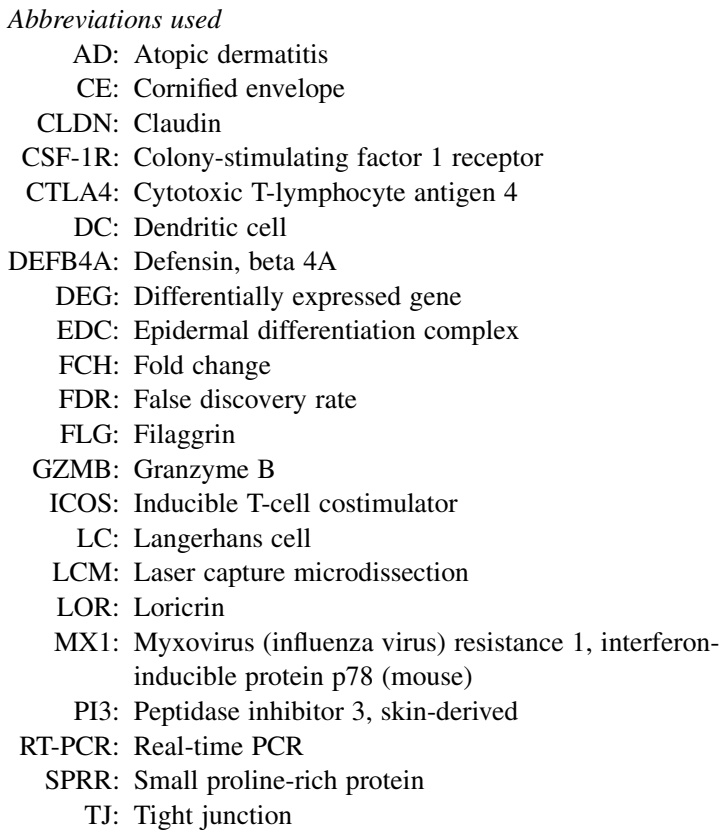

under direct microscopic visualization to study alterations in disease states. ${ }^{15}$ Our group has previously demonstrated that epidermal and dermal separation of lesional and nonlesional samples from patients with psoriasis and normal samples by using LCM complemented by microarrays largely increases the detection of low-abundance genes compared with whole-tissue analyses. $^{16,17}$ Despite the pathogenic relevance of separating the epidermal and dermal compartments, such studies are unavailable in patients with AD.

In this study we sought to determine the molecular phenotypes of the epidermal and dermal compartments of lesional and nonlesional AD skin (compared with skin from healthy subjects). Overall, our results (1) enlarged the AD transcriptome; (2) detected low-abundance genes (which are usually present at less than detection levels on whole-tissue microarrays [eg, IL22 and thymic stromal lymphopoietin [TSLP]); and (3) identified novel immune and barrier genes (ie, IL34, claudin 4 [CLDN4], and $C L D N 8$ ) and suggested possible cellular sources of immune markers (ie, CCR7).

\section{METHODS}

\section{Skin samples}

Paired nonlesional and lesional AD skin biopsy specimens were collected from 5 patients with moderate-to-severe chronic AD ( 3 male and 2 female patients; age, 27-59 years; mean age, 39.4 years; see Table E1 in this article's Online Repository at www.jacionline.org) under institutional review board-approved protocols. Normal skin samples from healthy volunteers $(\mathrm{n}=10)$ that had been collected for a prior LCM publication ${ }^{18}$ were also included. Paired epidermal, dermal, and full-thickness lesional and nonlesional samples were used for RT-PCR and microarray analysis ( $\mathrm{n}=5$ in each group). Lesional and nonlesional expression values were compared with 10 epidermal, 6 dermal, and 6 bulk corresponding normal samples. For RT-PCR confirmation, 3 normal paired epidermal and dermal samples were used because of the limited available mRNA.

\section{Slide preparation and LCM}

LCM was performed according to the manufacturer's protocol for the CellCut system (Molecular Machines \& Industries, Haslett, Mich; see the Methods section in this article's Online Repository at www.jacionline.org).

\section{RNA extraction}

Total RNA was extracted with the RNeasy Micro Kit (Qiagen, Valencia, Calif), according to the manufacturer's protocol, with on-column DNase digestion.

\section{Sample preparation for gene chip analysis}

Target amplification and labeling was performed according to the Affymetrix protocols for 2-cycle cDNA synthesis by using Affymetrix Human Genome U133 Plus 2.0 arrays (Affymetrix, Santa Clara, Calif), as previously reported. ${ }^{16}$

\section{Sample preparation for quantitative RT-PCR}

Reverse transcription to cDNA from RNA of LCM samples was carried out by using the High Capacity cDNA Reverse Transcription kit (Applied Biosystems, Foster City, Calif), cDNA was amplified with TaqMan PreAmp Master Mix (Applied Biosystems), and the preamplified cDNA product was analyzed with TaqMan Gene Expression Master Mix, according to the manufacturer's instructions. The RT-PCRs for each assay were run in triplicate, and all data were normalized to human acidic ribosomal protein P0. The primers and probes used in this study are listed in Table E2 in this article's Online Repository at www.jacionline.org.

\section{Immunohistochemistry and immunofluorescence}

Immunohistochemistry and immunofluorescence were performed on frozen skin sections, as previously described. ${ }^{19}$ The antibodies used in this study are listed in Table E3 in this article's Online Repository at www. jacionline.org.

\section{Statistical analysis}

Preprocessing and statistical analysis of microarray data were conducted with R (R-project.org) and Bioconductor software packages. ${ }^{20}$ Full details of the pipeline and downstream analysis are described in the Methods section in this article's Online Repository. Succinctly, the Harshlight package ${ }^{21}$ was used to quality control the images, and expression values were obtained by using the GCRMA algorithm. ${ }^{22}$ Batch adjustments were carried out with the ComBat algorithm, and mixed-effect models in the limma package were used to model differential expression. ${ }^{23-25}$ Genes with a false discovery rate (FDR) of less than 0.05 and a fold change of greater than 2 were considered significantly differentially expressed. Similar models were used to analyze $\log _{2}$-transformed values of normalized RT-PCR data.

\section{RESULTS \\ LCM localizes genes selectively expressed in the dermis and epidermis}

We performed LCM to collect epidermal and dermal (papillary, reticular, and inflammatory aggregate) cells in frozen sections of lesional, nonlesional, and normal skin samples, as shown in Fig 1, A. Microarray profiling of lesional, nonlesional, and normal epidermal and dermal tissues was performed with Affymetrix HGU133Plus2.0 microarrays to define the epidermal and dermal transcriptomes. A heat map of epidermis- and dermis-specific genes shows clear separation of differentially expressed genes (DEGs) localized to the epidermis and dermis across lesional, nonlesional, and normal samples (see Fig E1 in this article's 
LS

A

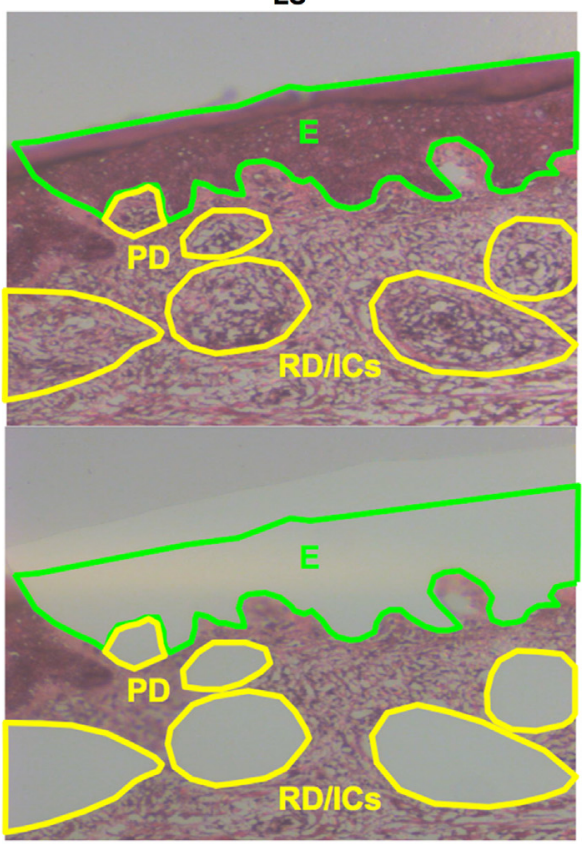

NL

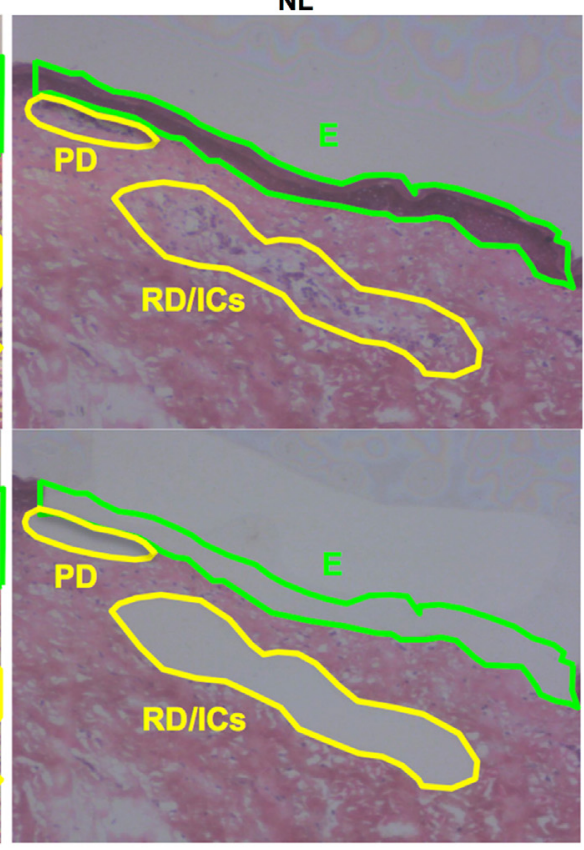

C

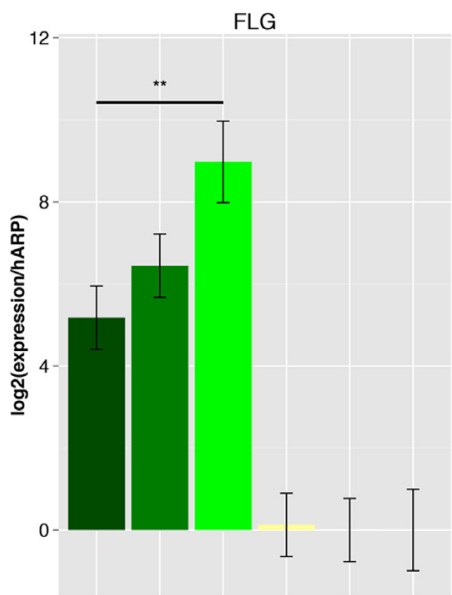

LS-E NL-E N-E LS-D NL-D N-D

D

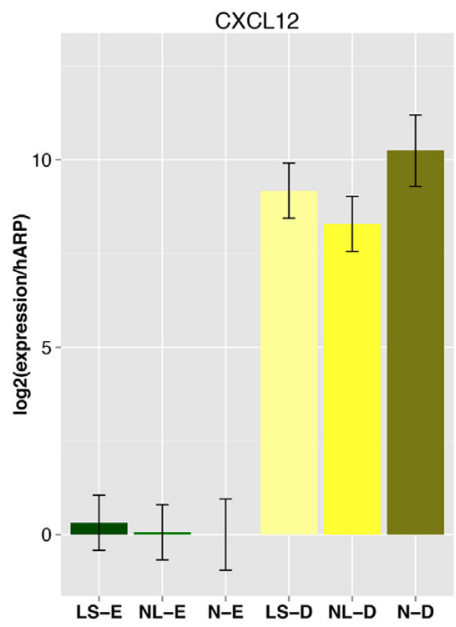

LS-E NL-E N-E LS-D NL-D N-D

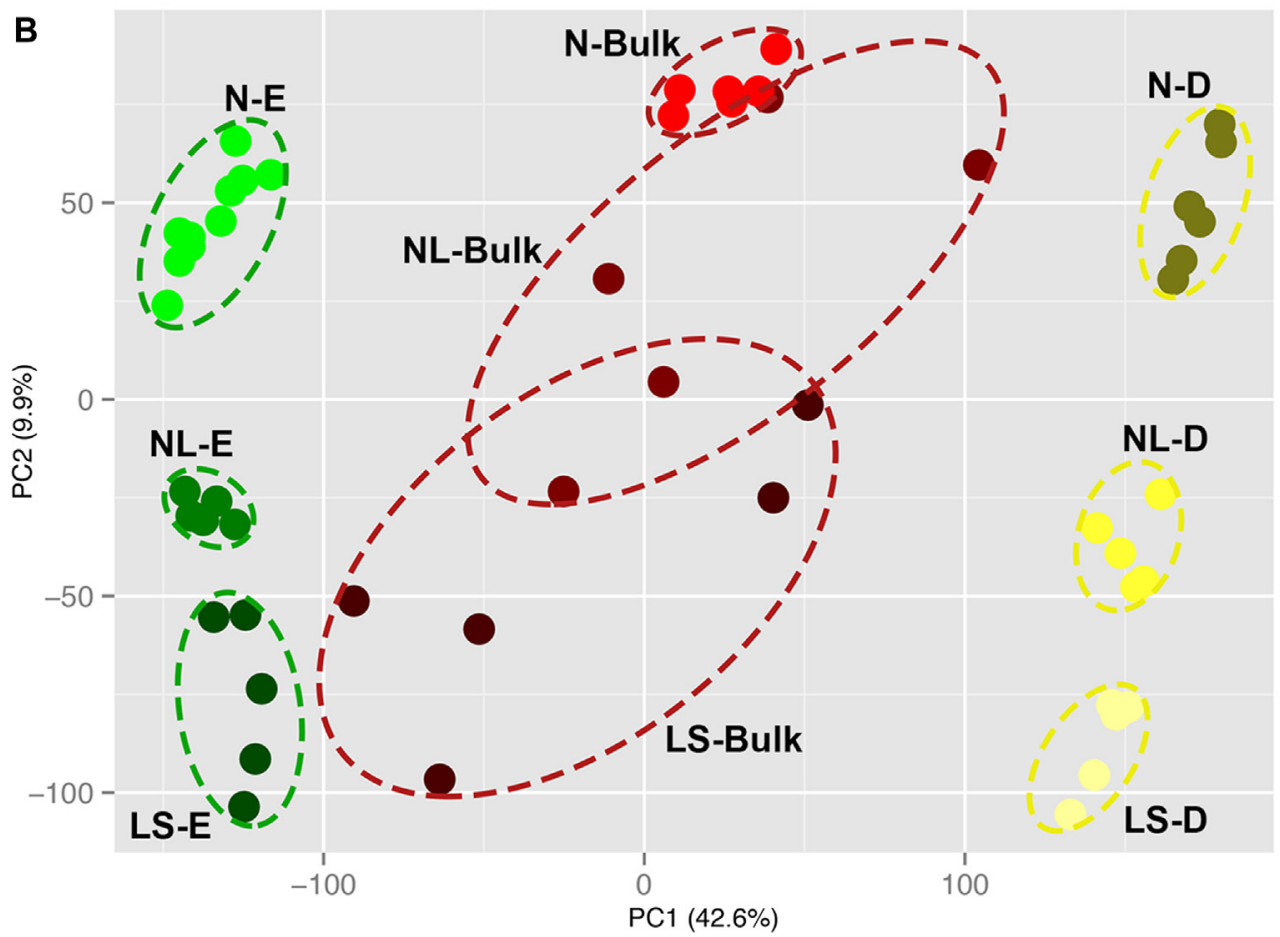

FIG 1. A, Representative hematoxylin and eosin staining of lesional (LS) and nonlesional (NL) AD skin. LCM was performed in the indicated zones (green: epidermis [E]; yellow. papillary dermis [PD], reticular dermis $[R D]$, and inflammatory aggregates $[I C s]$. B, Principal component $[P C]$ analysis plot showing clear separation of groups. $\mathbf{C}$ and $\mathbf{D}$, mRNA expression of $F L G(F i g 1, C)$ and $C X C L 12$ (Fig 1,D) in the epidermis $(E)$ and dermis $(D)$ of lesional (LS) and nonlesional (NL) AD and normal $(N)$ skin. Values are presented as means \pm SDs. ${ }^{* *} P<.01$.

Online Repository at www.jacionline.org). A principal component analysis of expression values illustrates the lack of outliers and that samples cluster in accordance with tissue type (Fig 1, B). Markers primarily considered dermal (CXCL12, $C D 93$, and collagens [COL1A2 and COL6A3]) were highly represented in the dermis (see Tables $\mathrm{E} 4, A$, and $\mathrm{E} 5, A$, in this article's Online Repository at www.jacionline.org), and epidermal markers (filaggrin [FLG], loricrin [LOR], late cornified envelope [LCE], and small proline-rich proteins [SPRRs]) were localized to the epidermis, which is consistent with the clear 
A

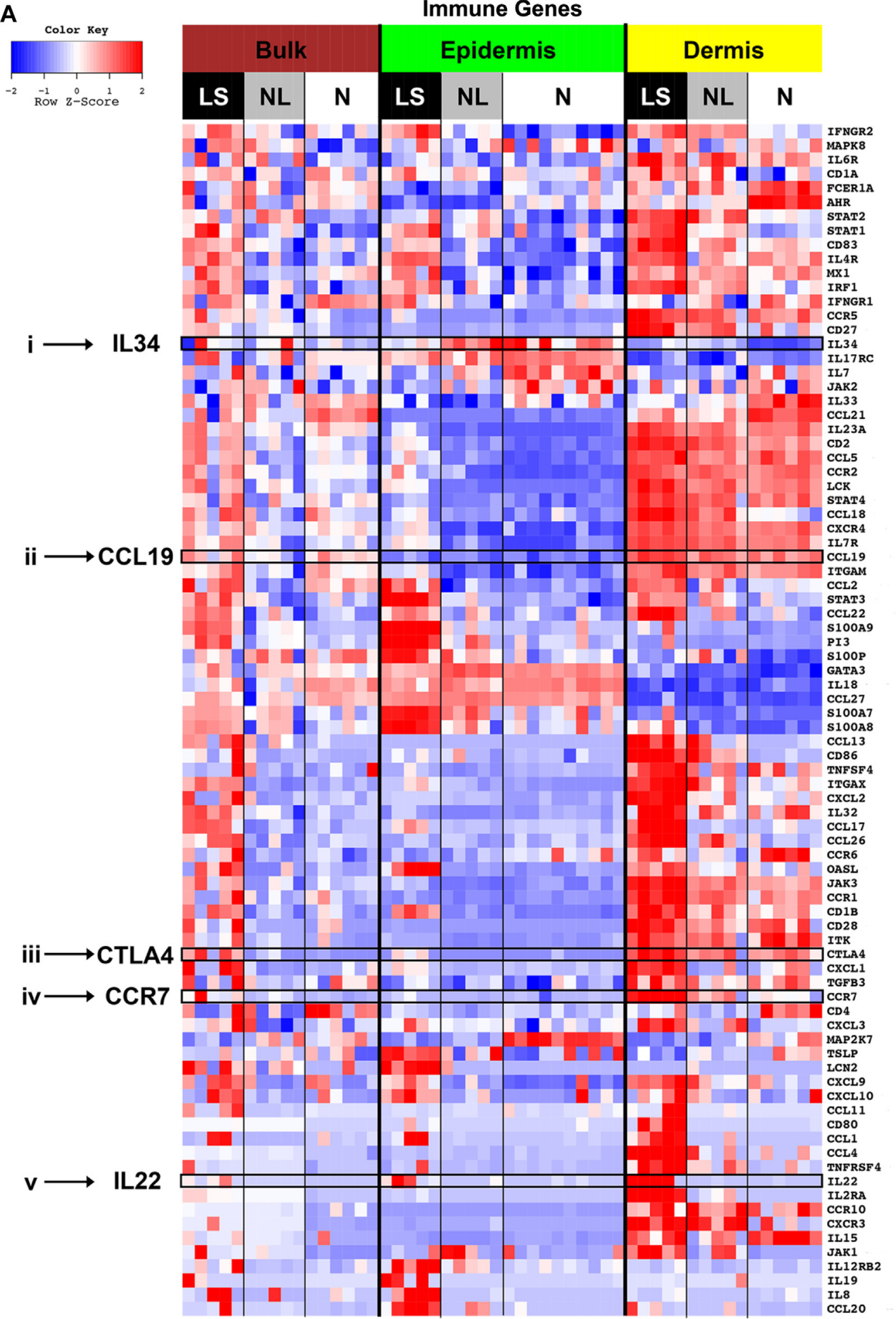


A

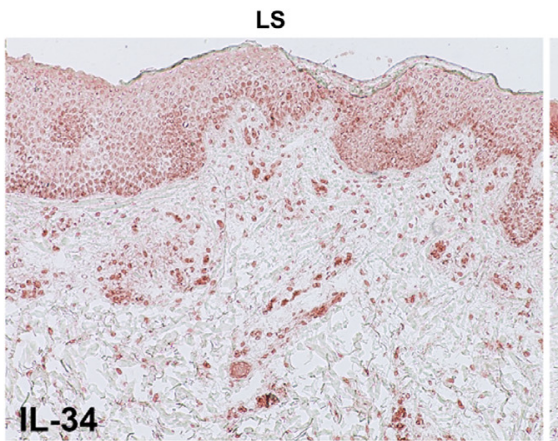

B

LS

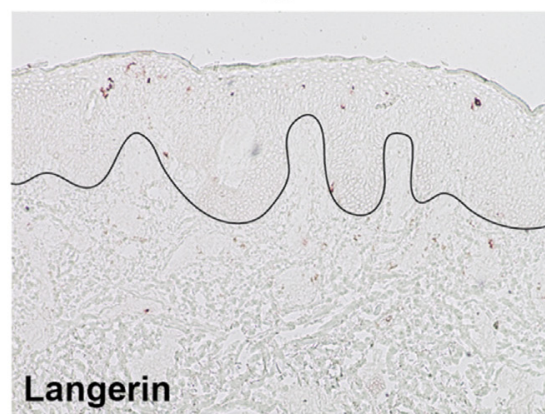

C
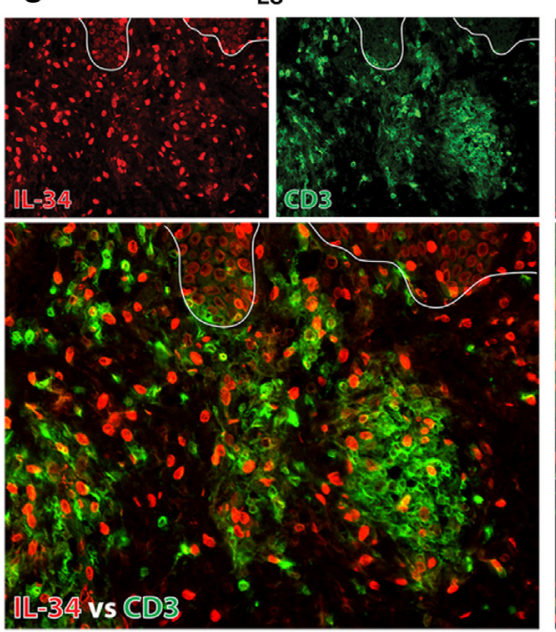

D
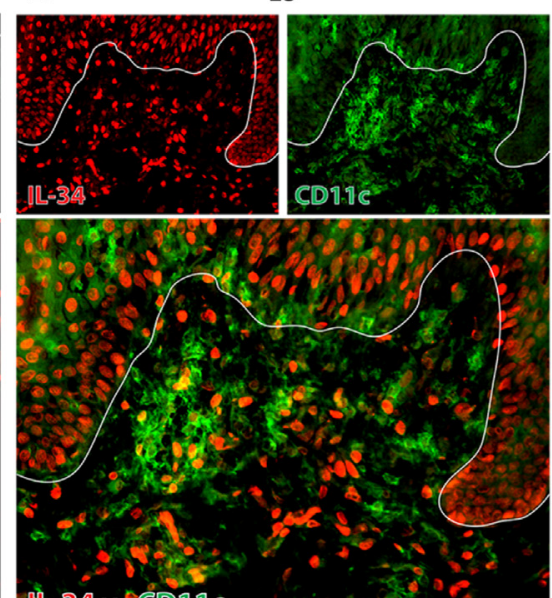

IL-34 vs CDD1C

CSF1R

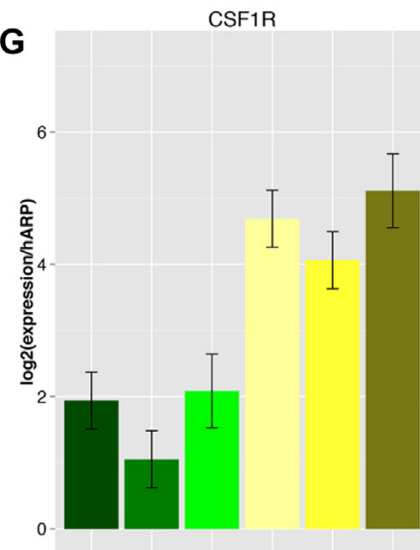

LS-E NL-E N-E LS-D NL-D N-D

FIG 3. A and B, Representative staining of IL-34 (Fig 3, A) and langerin (Fig 3, B) in lesional (LS) and nonlesional (NL) AD and normal (N) skin. C-E, Representative double immunofluorescence for coexpression of IL-34 (red) versus CD3 (green; Fig 3, C), CD11c (green; Fig 3, D), and CD163 (green; Fig 3, E) in lesional (LS) skin. $\mathbf{F}$ and $\mathbf{G}$, mRNA expression of $I L 34$ (Fig 3,F) and its receptor, CSF1R (Fig 3, G), in the epidermis (E) and dermis $(D)$ of lesional $(L S)$, nonlesional $(N L)$, and normal $(N)$ skin. Values are presented as means \pm SDs. ${ }^{* *} P<.01$. 
A

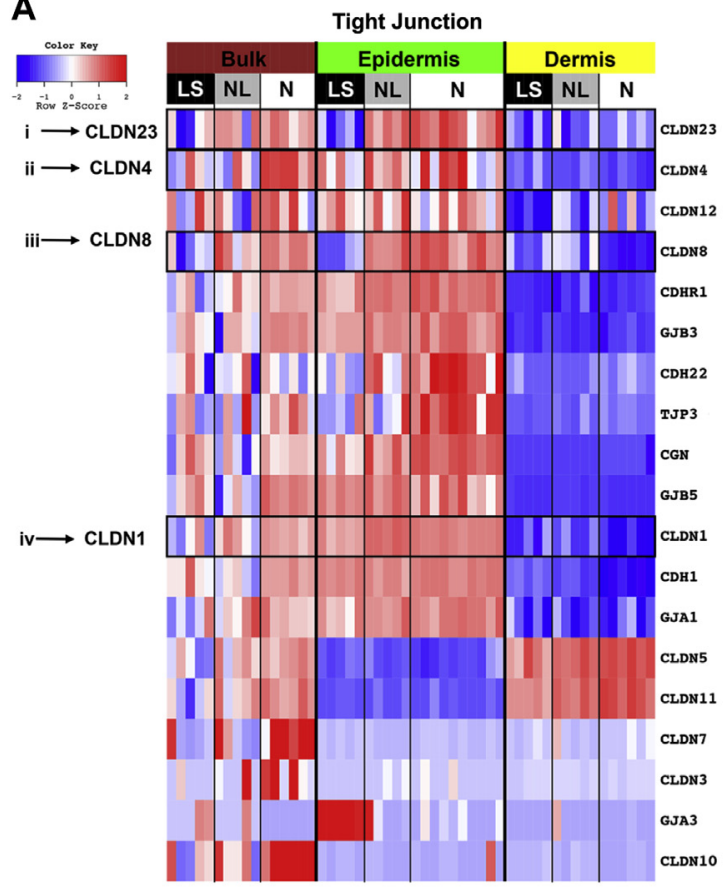

B
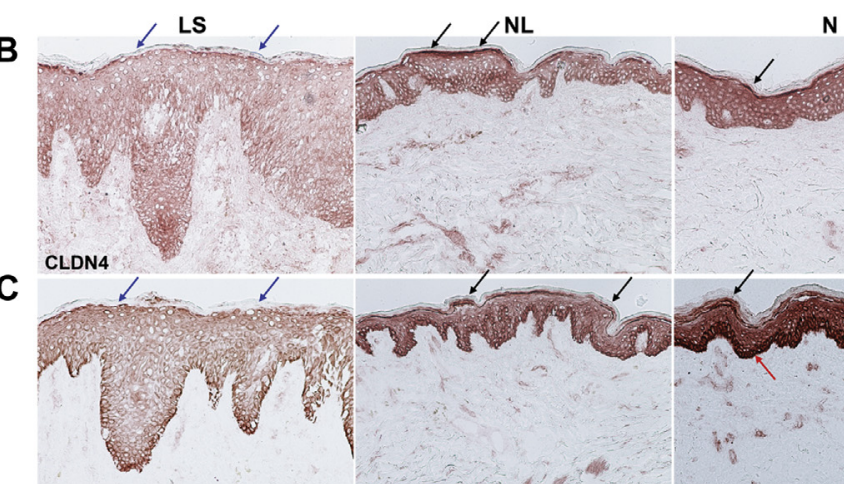

N

D

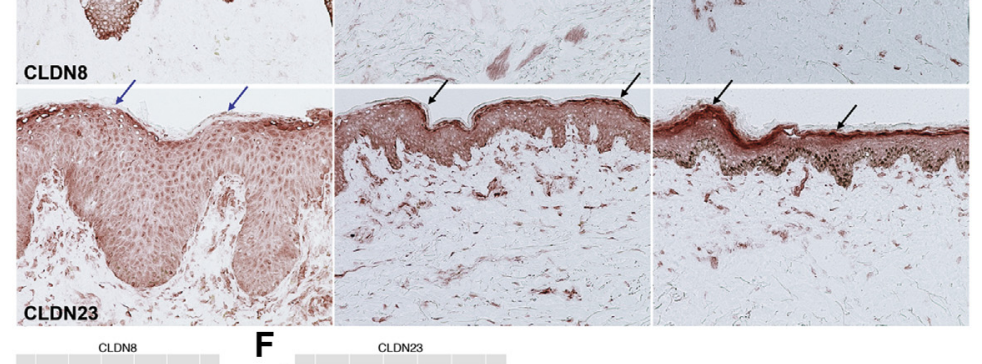

E

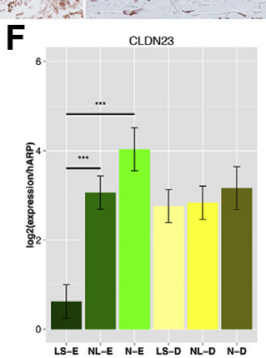

FIG 4. A, Heat map of barrier-related gene FCHs comparing lesional (LS), nonlesional (NL), and normal (N) skin in bulk, epidermal, and dermal specimens $(n=5 A D$ specimens and $n=6,10$, and 6 normal bulk, epidermis, and dermis specimens, respectively). B-D, Representative CLDN4 (Fig 4, B), CLDN8 (Fig 4, C), and CLDN23 (Fig 4,D) staining in lesional (LS), nonlesional (NL), and normal (N) skin. Arrows, Increased stratum corneum (black), decreased lesional stratum corneum (blue), and increased normal skin basal layer (red) staining. E and F, Expression of CLDN8 (Fig 4, E) and CLDN23 (Fig 4, F) in the epidermis (E) and dermis $(D)$ of lesional (LS), nonlesional (NL), and normal $(N)$ skin. Values are presented as means \pm SDs. ${ }^{*} P<.05$ and ${ }^{* *} P<.001$.

epidermal-dermal separation (see Tables E4, $B$, and E5, B). The accuracy of the LCM separation was validated by using RTPCR, with greater than 69.7-fold and greater than 846.2-fold enrichment of the mRNA expressions of FLG and CXCL12 in the epidermis versus dermis, respectively, in normal tissues (Fig 1, $C$ and $D$ ).

\section{LCM enlarges the AD transcriptome and increases detection of low-abundance immune genes on microarrays}

To characterize the AD phenotype within each compartment, we defined the lesional epidermal and dermal transcriptomes as the set of DEGs between lesional and nonlesional tissue within each compartment, respectively, by using the classical criteria of an FCH of greater than 2.0 and an FDR of less than 0.05. Combining the epidermal and dermal transcriptomes, many more compartment-specific DEGs were identified (860 upregulated and 495 downregulated, see Fig E2 in this article's Online Repository at www.jacionline.org), adding 674 upregulated and 405 downregulated DEGs to the recently defined AD transcriptome. ${ }^{11,12,26}$ The bulk transcriptome consists of 710 upregulated and 487 downregulated DEGs, whereas the LCM epidermal transcriptome contains 566 upregulated and 268 downregulated DEGs, and the LCM dermal transcriptome contains 330 upregulated and 244 downregulated DEGs. Little overlap was observed between the epidermal and dermal transcriptomes (only 36 upregulated and 17 downregulated DEGs were detected in both).

Overall, the top 25 upregulated and downregulated genes in each of the epidermal and dermal lesional transcriptomes included many genes that have been shown to contribute to the AD phenotype (see Tables E6 and E7 in this article's Online Repository at www.jacionline.org). ${ }^{11,12}$ The top 25 upregulated genes in the lesional epidermal transcriptome consisted of proliferation-related (KRT6A, KRT6B, and KRT16), epidermal differentiation complex (EDC; S100 genes), inflammatory (matrix metalloproteinase 12 [MMP12]), and antimicrobial peptide-derived (defensin, beta 4A [DEFB4A] and peptidase inhibitor 3, skin-derived [PI3]/Elafin) genes (see Table E6, A). The top 25 upregulated genes in the lesional dermal transcriptome included T-cell activation (granzyme $\mathrm{B}[G Z M B]$ and IL-2 receptor $\alpha[I L 2 R A]$ ), $\mathrm{T}_{\mathrm{H}}$ 2-related (CCL17, CCL22, and CCL26), $\mathrm{T}_{\mathrm{H}} 22$ / $\mathrm{T}_{\mathrm{H}}$ 17-related (IL22, S100A8, CXCL1, and CXCL2), and collagen (COL6A6, COL6A5, and COL4A3) genes (see Table E6, B). IL22 expression was significantly increased in the lesional dermal transcriptome $(12.46 \mathrm{FCHs}$ and FDR $<0.001)$, whereas it was present 


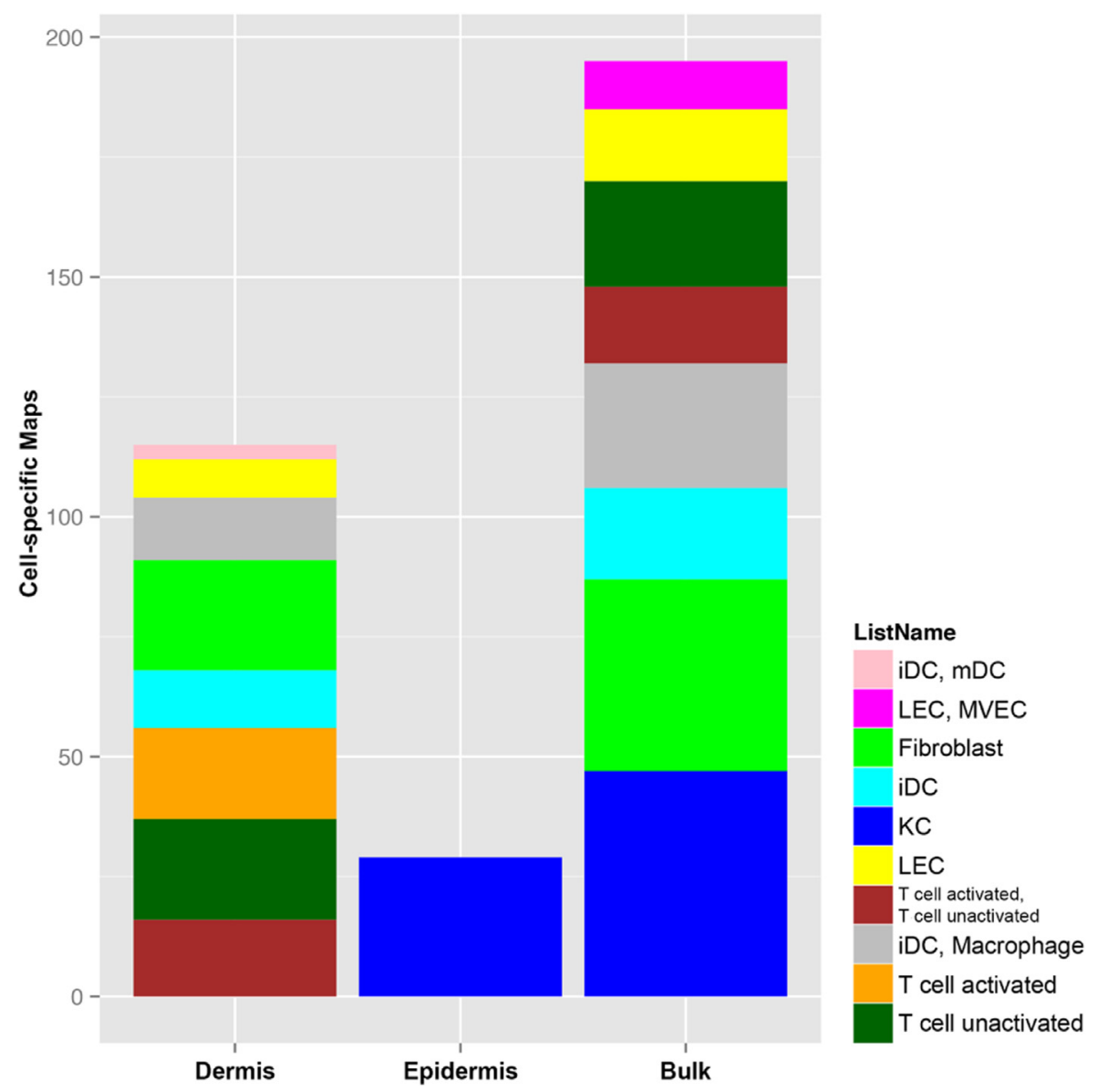

FIG 5. The cell-specific map of the LCM epidermal and dermal transcriptomes and bulk transcriptome. The cellular source of the epidermal transcriptome was mainly keratinocytes, whereas activated $\mathrm{T}$ cells were only significantly enriched in the LCM dermal transcriptome. $i D C$, Immature dendritic cell; $K C$, keratinocyte; $L E C$, lymphatic endothelial cell; $m D C$, mature dendritic cell; $M V E C$, microvascular endothelial cell.

at less than detection levels in previous reports, ${ }^{11,12,26}$ indicating that LCM is a useful method to increase the sensitivity of detecting low-abundance genes.

Key inflammatory and barrier genes were uniquely detected in the LCM epidermal (see Table E8, $A$, in this article's Online Repository at www.jacionline.org) and dermal (see Table E8, B) transcriptomes. The unique lesional epidermal transcriptome included IL-17-related (DEFB4A and CCL2O) and inflammatory (CXCR4, signal transducer and activator of transcription 3 [STAT3], and IL8) genes (see Table E8, A). Key $\mathrm{T}_{\mathrm{H}} 2$ (CCL17, CCL22, and CCL26) and $\mathrm{T}_{\mathrm{H}} 22$ (IL22) markers and $\mathrm{T}$-cell migration/activation markers (CCR7 and inducible T-cell costimulator $[I C O S]$ ) were found within the unique lesional dermal transcriptome (see Table E8, B). Although the LCM approach detected an appreciably larger number of genes within bulk tissues, there was a subset of DEGs that were not identified in the corresponding epidermal and dermal transcriptomes (429 upregulated and 381 downregulated genes; Fig 2 and see Table $\mathrm{E} 8, C)$. A deeper analysis of these data suggests that most of this discrepancy is created by increased or decreased gene abundance because of differing contributions of the epidermis and dermis in lesional versus nonlesional skin (see the Results section and Fig E3 in this article's Online Repository at www. jacionline.org).
We further explored the compartmental distribution of immune DEGs in lesional, nonlesional, and normal skin using our previously curated and reported immune gene subset, ${ }^{19,26,27}$ as shown in a heat map (Fig 2, A, and see Table E9 in this article's Online Repository at www.jacionline.org). Among significantly upregulated genes in the lesional dermal transcriptome were T-cell (cytotoxic T-lymphocyte antigen 4 [CTLA4] and CD28), DC (integrin $\alpha \mathrm{X}$, complement component 3 receptor 4 subunit [ITGAX]/CD11c and CDIA), lymphoid-organizing chemokine (CCR7 and its ligands [CCL19 and CCL21]), and $\mathrm{T}_{\mathrm{H}} 2$-related (CCL11, CCL13, CCL17, CCL22, CCL26, TNF receptor superfamily, member 4 [TNFRSF4/OX40], and IL4R) genes. Significant increases in $\mathrm{T}_{\mathrm{H}}$ 2-related $(I L 7 R), \mathrm{T}_{\mathrm{H}}$ 17-related $(P I 3$, lipocalin 2 [LCN2], CCL20, and STAT3), $\mathrm{T}_{\mathrm{H}} 22 / \mathrm{T}_{\mathrm{H}} 17$-related (IL22, S100A8, and S100A9), and $\mathrm{T}_{\mathrm{H}} 1$-related (2'-5'-oligoadenylate synthetase-like [OASL], myxovirus [influenza virus] resistance 1 , interferon-inducible protein p78 [mouse] [MX1], IL12RB2, IFN- $\gamma$ receptor 2 [IFNGR2], and interferon regulatory factor $1[I R F 1]$ ) products were found. Using RT-PCR, we validated the primarily dermal mRNA expression of selected markers, including IL22, CTLA4, CCR7, and CCL19. High IL22 mRNA levels were characteristic of only lesional skin (Fig 2, B-E). A list of DEGs in all comparisons is presented in Table E8. 


\section{IL-34: A novel cytokine identified by means of LCM}

IL-34 was significantly downregulated in lesional compared with both nonlesional and normal epidermis (Fig 2, A, and see Table E9). IL-34 is a recently identified cytokine in mice and normal human $\operatorname{skin}^{28}$ and is suggested to regulate LC differentiation in steady states. It has been identified as an alternative ligand to the colony-stimulating factor 1 receptor (CSF-1R), which has been shown to be expressed in epidermal LCs, dermal monocytes, and DCs. ${ }^{29}$ IL-34 has not been previously reported in human skin diseases. We performed immunohistochemical staining of IL-34, which showed stronger epidermal staining in normal and nonlesional skin compared with lesional skin and stronger dermal staining in lesional skin (Fig 3, A).

Because IL-34 was reported to induce LC differentiation only in steady states, ${ }^{29}$ langerin (CD207) staining for LCs was performed. Many fewer LCs were detected in lesional compared with both nonlesional and normal skin (Fig 3, B). To identify the cellular distribution of IL-34 expression in the dermis, we performed double immunofluorescence for IL-34 (red) with $\mathrm{CD}^{+} / \mathrm{T}$ cells (green; see Fig E4, A, in this article's Online Repository at www.jacionline.org), CD11c ${ }^{+} / \mathrm{DCs}$ (green; see Fig E4, B), and $\mathrm{CD}_{163}{ }^{+}$/macrophages (green; see Fig E4, C) in normal, nonlesional, and lesional skin. Few double-positive IL- $34^{+} / \mathrm{CD}^{+}{ }^{+}$cells were found. Many IL- $34^{+}$cells colocalized with $\mathrm{CD}_{11 \mathrm{c}^{+}}$and $\mathrm{CD} 63^{+}$cells (Fig 3, $C-E$ ). Thus IL-34 in the dermis is preferentially expressed by myeloid DCs and macrophages.

We validated the lower expression of IL-34 in lesional epidermis using RT-PCR, with significantly lower IL34 mRNA expression observed in lesional versus both nonlesional and normal epidermis and with slightly lower dermal mRNA expression (Fig 3,F). We also measured CSF1R mRNA expression, which was significantly higher in the dermis, regardless of tissue (Fig 3, $G$ ).

\section{LCM highlights tight junction defects in patients with AD}

Because defective barrier function is a hallmark of $\mathrm{AD},{ }^{1}$ we evaluated for EDC and cornified envelope (CE) markers. A heat map of EDC and CE genes (including S100A genes) is shown in Fig E5 in this article's Online Repository at www.jacionline. org. The majority of S100 genes (S100A8/S100A9/S100A12/ S100A13), IL-17-induced PI3/Elafin, and SPRRs (SPRRIA) showed a primarily epidermal expression and higher expression in lesional versus both nonlesional and normal skin (FDR < 0.001 for most markers), whereas a few S100 genes (S100A4/ S100A6/S100A10) showed predominantly dermal lesional expression. As previously reported in bulk tissues, ${ }^{26}$ the differentiation genes ( $L C E, L O R, F L G$, and periplakin [PPL]) showed decreased expression in lesional versus nonlesional and normal epidermis (see Fig E5 and Table E10 in this article's Online Repository at www.jacionline.org). Claudins, another crucial barrier component, are essential for tight junction (TJ) formation. Twenty-three claudins have been identified in human subjects; however, their contribution to $\mathrm{AD}$ is not fully defined. ${ }^{30,31}$ To gain insight into which claudins are expressed in patients with $\mathrm{AD}$, we evaluated a subset of $\mathrm{TJ}$ genes, the expression profiles of which are visualized in a heat map (Fig 4, $A$, and see Table E11 in this article's Online Repository at www.jacionline.org). Downregulation of claudins 1 and 23 was observed in lesional versus nonlesional and normal epidermis, as well as downregulation of claudins 4 and 8 , which were previously unreported in patients with $\mathrm{AD}(\mathrm{FDR}<0.001) .{ }^{32} \mathrm{We}$ also detected dermal claudins (5 and 11), expression of which was significantly downregulated in the lesional versus normal skin comparison, which was previously not reported in patients with AD.

The differential expression of claudin genes in the epidermal and dermal compartments was validated by using immunohistochemistry and RT-PCR. CLDN4, CLDN8, and CLDN23 stained all epidermal layers, except the stratum corneum (Fig 4, $B-D$ ), with highly attenuated staining in lesional versus both nonlesional and normal epidermis. The reduced staining was particularly evident in the granular layer. CLDN8 showed increased intensity of basal layer staining in normal epidermis (Fig 4, $C$ ). The mRNA expression of $C L D N 8$ and $C L D N 23$ was also significantly reduced in lesional epidermis $(P<.05$; Fig $4, E$ and $F)$.

\section{Markers associated with activated $T$ cells and other inflammatory cells are enriched by using LCM}

Using individual cell-culture expression data (keratinocytes, fibroblasts, activated and unactivated T cells, DCs, and LCs), ${ }^{33-35}$ we explored the distribution of DEGs identified in epidermal, dermal, and bulk AD transcriptomes ${ }^{27}$ using a Gene Set Enrichment Analysis. Whereas only the keratinocyte gene subset was enriched in the epidermal transcriptome (Fig 5 and see Table $\mathrm{E} 12, A$, in this article's Online Repository at www.jacionline.org), the dermal transcriptome showed enrichment of key inflammatory subsets, including activated and unactivated $\mathrm{T}$ cells, various DC subsets (immature and mature DCs), macrophages, fibroblasts, and lymphatic endothelial cells (Fig 5 and see Table E12, B). The bulk transcriptome showed a significant enrichment for keratinocytes, fibroblasts, and inflammatory subsets, with no enrichment for activated $\mathrm{T}$ cells and mature DCs (Fig 5 and see Table E12, C).

The Gene Set Enrichment Analysis also linked key mediators (IL22, TNF superfamily, member 4 [TNFSF4/OX40L], and CTLA4) detected in the dermal transcriptome to the gene signature of activated T cells (see Table E12, B). Among the genes found to be associated with both activated and unactivated T cells in the dermal transcriptome were GZMB, ICOS, and CD27.

CCR7, which is associated with T-cell trafficking and cutaneous lymphoid aggregates, ${ }^{36,37}$ was shown to be expressed by various cells types, including T cells and DCs. To investigate the predominant cellular expression of CCR7, we performed double immunofluorescence staining for $\mathrm{CD}^{+}{ }^{+} \mathrm{T}$ cells (green) or CD11 ${ }^{+}$myeloid DCs (green) with CCR7 (red) in lesional, nonlesional, and normal skin. CCR7 colocalized mainly with CD11c, showing increased expression in lesional versus nonlesional and normal dermis and some colocalization with CD3, primarily in lesional dermis (see Fig E6 in this article's Online Repository at www.jacionline.org). Thus CCR7 is preferentially expressed by myeloid DCs rather than T cells.

\section{DISCUSSION}

Evolving disease concepts associate the AD phenotype with barrier and immune abnormalities. ${ }^{1,2}$ In this model altered proliferation and differentiation of keratinocytes result from 
cytokines derived from distinct T-cell subsets. ${ }^{2,12}$ It is important to understand the relative contributions of the epidermal and dermal compartments in creating the abnormal lesional phenotype to fully understand the pathogenic mechanisms driving $\mathrm{AD}^{26}$

This is the first report that establishes robust epidermal and dermal genomic signatures of lesional and nonlesional $\mathrm{AD}$ and normal skin compared with corresponding whole-tissue fingerprinting. Using bulk skin, we have associated the AD phenotype with $\mathrm{T}_{\mathrm{H}} 2 / \mathrm{T}_{\mathrm{H}} 22$ immune activation and abnormal epidermal differentiation. ${ }^{11-14}$ However, in bulk genomic profiling we could not determine which compartments are responsible for individual gene expression or locate their cellular sources. ${ }^{4,12,27}$ Furthermore, because of a dilution effect in bulk tissues, which are composed of functionally heterogeneous cells, many genes linked to $\mathrm{AD}$ pathogenesis are present at less than the detection level on microarrays. ${ }^{11,12,26,27}$ Additionally, because a large subset of genes are expressed in either the epidermis or dermis only, some of the measured differential gene expression between lesional and nonlesional skin appears to be an artifact of the unequal contribution of epidermal expansion in lesional skin rather than because of true changes in gene expression at the cellular level. The previous disease model for AD, which has relied on bulk tissue genomic profiling, is limited by the inability to distinguish between these differences. A large proportion of DEGs found only in bulk tissue seem to derive from unequal contributions of lesional epidermis and dermis compared with nonlesional epidermis and dermis. Overall, we believe that the differences in cellular physiology created by AD are best detected in the LCM-generated DEGs.

Using the LCM method followed by genomic and cellular studies, we have identified a largely increased AD transcriptome, with an additional 674 upregulated and 405 downregulated genes compared with prior reports. ${ }^{1,12,26,27}$ By separating the 2 compartments, we have also identified key immune and barrier markers that are usually undetectable on arrays (ie, IL-22 and TSLP) and obtained more robust genomic differences for most immune genes when comparing lesional, nonlesional, and normal tissues. Through this approach and previously acquired pathways and cell-specific genomic maps, ${ }^{11-14,26,27}$ we have localized many immune and barrier genes to the epidermis or dermis (or both), leading to a deeper understanding of inflammatory circuits and the cellular subset involved in creating the AD phenotype. For example, dermal expression of CCL19 and CCL21 likely attracts $\mathrm{CCR}^{+} / \mathrm{CD} 11 \mathrm{c}^{+} \mathrm{DCs}$ and might organize lymphoid tissue in the dermis. Our LCM and genomic approach also led to enrichment of gene products associated with activated $\mathrm{T}$ cells and inflammatory DCs that play a role in effector responses in patients with $\mathrm{AD} .^{37}$

Our data are the first to identify IL-34, the newly identified cytokine in mice models, and its receptor, CSF-1R, in a human skin disease. The mouse studies demonstrated a critical role for IL-34 in differentiation and proliferation of LCs in the epidermis during steady states, whereas repopulation of LCs in inflammatory states was independent of IL-34. ${ }^{28}$ Although IL-34 is mainly produced by keratinocytes, its receptor, CSF-1R, originates from dermal macrophages and mononuclear cells. ${ }^{29}$ Our data show decreased epidermal expression of IL-34 in lesional epidermis compared with that seen in both nonlesional $\mathrm{AD}$ and normal epidermis (Fig 3, $B$ and $C$ ). This confirms the role of IL-34 in the maintenance phase, corresponding to prior mouse data. ${ }^{28}$
Thus the IL-34 cytokine might function as a negative regulator, and its induction in nonlesional skin might inhibit the propagation of the inflammatory cascade toward development of active skin lesions. Additionally, we found that IL-34 expression in lesional dermis colocalizes most commonly with myeloid DCs and macrophages. Future studies are needed to evaluate the possible functions of the IL-34/CSF-1R cytokine-receptor complex in background and diseased $\mathrm{AD}$ skin and whether strategies of increasing levels of this cytokine might be able to prevent development of skin lesions.

Separating the 2 compartments, we also identified 2 novel barrier genes, $C L D N 4$ and $C L D N 8$, for the first time in the AD transcriptome. Claudins are pivotal for TJ formation. ${ }^{38}$ Prior mouse and human skin equivalent models demonstrated that CLDN4 colocalized with CLDN1 to the epidermal granular layer, ${ }^{39,40}$ whereas CLDN8 was previously shown in human kidneys and intestines. ${ }^{41,42}$ Nevertheless, our knowledge of claudins and their involvement in the barrier alterations seen in patients with AD remains incomplete. ${ }^{29}$ De Benedetto et $\mathrm{al}^{32}$ reported that $C L D N 1$ and $C L D N 23$ show significantly reduced expression in nonlesional AD skin compared with that seen in healthy skin. In our study CLDN8 and CLDN23 showed significantly reduced expression in lesional compared with nonlesional and normal epidermis by using microarrays (Fig 4, $A$, and see Table E11), with respective fainter epidermal staining of CLDN4, CLDN8, and CLDN23 in lesional versus nonlesional $\mathrm{AD}$ and normal epidermis (Fig 4, B and D). Claudin 11 was identified as a novel dermal claudin, which was previously shown in other tissues (brain, testis, and cochlea). ${ }^{43-45}$ Future experiments are needed to investigate the function of the newly identified CLDN4 and CLDN8, but together with CLDN1 and CLDN23, these might contribute to the barrier defect associated with the AD phenotype.

Our study also had some limitations. First, LCM is a labor-intensive method, usually allowing analyses of a small number of samples, as in our study $(\mathrm{n}=5$ lesional and nonlesional specimens each and $\mathrm{n}=3$ normal samples separated into epidermis and dermis categories), possibly resulting in weaker statistical power, particularly for bulk tissue comparisons. Second, although our LCM approach detected novel barrier genes, the downregulation of key differentiation markers (ie, FLG, LOR, and involucrin [IVL]) in the lesional and nonlesional (vs normal) epidermal transcriptomes was not as impressive as in prior bulk data, ${ }^{13,26}$ which is contrary to the enrichment of FLG and $L O R$ in a prior normal skin epidermis LCM study, ${ }^{17,46}$ perhaps because of lower recovery of granular layer products using the epidermal-dermal separation approach. ${ }^{47}$ Third, even though the combined LCM epidermal and dermal transcriptomes were larger than the bulk tissue transcriptome, many genes were only detected in the bulk transcriptome (see Fig E2 and Table E8, $C$ ). This might be explained by (1) the unequal contribution of lesional epidermis and dermis compared with their counterparts; (2) possible inclusion of subcutaneous tissue in bulk biopsy specimens; (3) platform/technical issues, as described in a previous LCM article separating the epidermis and dermis from only 3 psoriatic (lesional and nonlesional) and 3 normal tissues ${ }^{16}$; and (4) restriction of this analysis to patients with severe $\mathrm{AD}$, in whom nonlesional skin has a more abnormal phenotype. ${ }^{26}$ Thus many genes might not pass defined thresholds of $\mathrm{FCH}$ and significance when comparing lesional and nonlesional skin. 
Our study establishes the utility of LCM in patients with AD to separate different skin compartments and cellular infiltrates. It provides complementary information to bulk analysis, allowing regional and/or cellular localization of key barrier or immune molecules, and enables detection of genes that are not usually detected on arrays because of the mixture of transcripts within the heterogeneous bulk tissue. ${ }^{48,49}$ LCM is particularly beneficial for $\mathrm{AD}$, in which a complex network of immune and barrier abnormalities results in the global phenotypes of active and "normal-appearing" skin, and dissecting the individual skin components and cells is crucial to unraveling their respective contributions to pathogenesis. Our combined LCM and genomic approach can be useful in future studies aimed at dissecting the relative roles of barrier versus immune activation of different $\mathrm{AD}$ phenotypes (ie, intrinsic and extrinsic $\mathrm{AD})^{11}$ and for dissecting therapeutic responses to various agents that are now in clinical trials for patients with $\mathrm{AD} .^{50}$

Clinical implications: Our approach can be useful to differentiate the ability of targeted treatments to reverse epidermal and immune alterations in AD skin.

\section{REFERENCES}

1. Bieber T. Atopic dermatitis. N Engl J Med 2008;358:1483-94.

2. Guttman-Yassky E, Nograles KE, Krueger JG. Contrasting pathogenesis of atopic dermatitis and psoriasis - part I: clinical and pathologic concepts. J Allergy Clin Immunol 2011;127:1110-8.

3. Boguniewicz M, Leung DY. Recent insights into atopic dermatitis and implications for management of infectious complications. J Allergy Clin Immunol 2010;125:4-15.

4. Nograles KE, Zaba LC, Shemer A, Fuentes-Duculan J, Cardinale I, Kikuchi T, et al. IL-22-producing "T22" T cells account for upregulated IL-22 in atopic dermatitis despite reduced IL-17-producing TH17 T cells. J Allergy Clin Immunol 2009;123:1244-52.

5. Ong PY, Leung DY. Immune dysregulation in atopic dermatitis. Curr Allergy Asthma Rep 2006;6:384-9.

6. Hatano Y, Terashi H, Arakawa S, Katagiri K. Interleukin-4 suppresses the enhancement of ceramide synthesis and cutaneous permeability barrier functions induced by tumor necrosis factor-alpha and interferon-gamma in human epidermis. J Invest Dermatol 2005;124:786-92.

7. Elias PM, Hatano Y, Williams ML. Basis for the barrier abnormality in atopic dermatitis: outside-inside-outside pathogenic mechanisms. J Allergy Clin Immunol 2008;121:1337-43.

8. Onoue A, Kabashima K, Kobayashi M, Mori T, Tokura Y. Induction of eosinophil- and Th2-attracting epidermal chemokines and cutaneous late-phase reaction in tape-stripped skin. Exp Dermatol 2009;18:1036-43.

9. Oyoshi MK, Larson RP, Ziegler SF, Geha RS. Mechanical injury polarizes skin dendritic cells to elicit a $\mathrm{T}(\mathrm{H}) 2$ response by inducing cutaneous thymic stromal lymphopoietin expression. J Allergy Clin Immunol 2010;126:976-84, e1-5.

10. Demehri S, Morimoto M, Holtzman MJ, Kopan R. Skin-derived TSLP triggers progression from epidermal-barrier defects to asthma. PLoS Biol 2009;7: e1000067.

11. Suarez-Farinas M, Dhingra N, Gittler J, Shemer A, Cardinale I, de Guzman Strong $\mathrm{C}$, et al. Intrinsic atopic dermatitis shows similar TH2 and higher TH17 immune activation compared with extrinsic atopic dermatitis. J Allergy Clin Immunol 2013;132:361-70.

12. Gittler JK, Shemer A, Suarez-Farinas M, Fuentes-Duculan J, Gulewicz KJ, Wang CQ, et al. Progressive activation of $\mathrm{T}(\mathrm{H}) 2 / \mathrm{T}(\mathrm{H}) 22$ cytokines and selective epidermal proteins characterizes acute and chronic atopic dermatitis. J Allergy Clin Immunol 2012;130:1344-54.

13. Guttman-Yassky E, Suarez-Farinas M, Chiricozzi A, Nograles KE, Shemer A, Fuentes-Duculan J, et al. Broad defects in epidermal cornification in atopic dermatitis identified through genomic analysis. J Allergy Clin Immunol 2009; 124:1235-44.e58

14. Guttman-Yassky E, Lowes MA, Fuentes-Duculan J, Whynot J, Novitskaya I, Cardinale I, et al. Major differences in inflammatory dendritic cells and their products distinguish atopic dermatitis from psoriasis. J Allergy Clin Immunol 2007;119:1210-7
15. Espina V, Wulfkuhle JD, Calvert VS, VanMeter A, Zhou W, Coukos G, et al. Laser-capture microdissection. Nat Protoc 2006;1:586-603.

16. Mitsui H, Suarez-Farinas M, Belkin DA, Levenkova N, Fuentes-Duculan J, Coats I, et al. Combined use of laser capture microdissection and cDNA microarray analysis identifies locally expressed disease-related genes in focal regions of psoriasis vulgaris skin lesions. J Invest Dermatol 2012;132: $1615-26$.

17. Gulati N, Krueger JG, Suarez-Farinas M, Mitsui H. Creation of differentiationspecific genomic maps of human epidermis through laser capture microdissection. J Invest Dermatol 2013;133:2640-2.

18. Ning H, Mitsui H, Wang C, Suarez-Farinas M, Gonazleaz J, Shah K, et al. Identification of anaplastic lymphoma kinase as a potential therapeutic target in basal cell carcinoma. Oncotarget 2013;4:2237-48.

19. Dhingra N, Shemer A, Correa da Rosa J, Rozenblit M, Suarez-Farinas M, Fuentes-Duculan J, et al. Molecular profiling of contact dermatitis skin identifies allergen-dependent differences in immune response. J Allergy Clin Immunol 2014;134:362-72.

20. Gentleman RC, Carey VJ, Bates DM, Bolstad B, Dettling M, Dudoit S, et al. Bioconductor: Open software development for computational biology and bioinformatics. Genome Biol 2004;5:R80.

21. Suarez-Farinas M, Pellegrino M, Wittkowski K, Magnasco M. Harshlight: a "corrective make-up" program for microarray chips. BMC Bioinformatics 2005;6:294.

22. Wu Z, Irizarry RA, Gentleman R, Martinez-Murillo F, Spencer F. A model-based background adjustment for oligonucleotide expression arrays. J Am Stat Assoc 2004;99:909-17.

23. Smyth GK. limma: linear models for microarray data. In Gentleman R, Carey VJ, Huber W, Irizarry RA, Dudoit S, editors. Bioinformatics and computational biology solutions using R and Bioconductor. New York: Springer-Verlag; 2005, pp. 397-420.

24. Boedigheimer MJ, Wolfinger RD, Bass MB, Bushel PR, Chou JW, Cooper M, et al. Sources of variation in baseline gene expression levels from toxicogenomics study control animals across multiple laboratories. BMC Genomics 2008; $12: 285$.

25. Johnson WE, Li C, Rabinovic A. Adjusting batch effects in microarray expression data using empirical bayes methods. Biostatistics 2007;8:118-27.

26. Suarez-Farinas M, Tintle SJ, Shemer A, Chiricozzi A, Nograles K, Cardinale I, et al. Nonlesional atopic dermatitis skin is characterized by broad terminal differentiation defects and variable immune abnormalities. J Allergy Clin Immunol 2011;127:954-64, e1-4.

27. Khattri S, Shemer A, Rozenblit M, Dhingra N, Czarnowicki T, Finney R, et al. Cyclosporine in patients with atopic dermatitis modulates activated inflammatory pathways and reverses epidermal pathology. J Allergy Clin Immunol 2014;133:1626-34.

28. Greter M, Lelios I, Pelczar P, Hoeffel G, Price J, Leboeuf M, et al. Stroma-derived interleukin-34 controls the development and maintenance of Langerhans cells and the maintenance of microglia. Immunity 2012;37:1050-60.

29. Sasmono RT, Oceandy D, Pollard JW, Tong W, Pavli P, Wainwright BJ, et al. A macrophage colony-stimulating factor receptor-green fluorescent protein transgene is expressed throughout the mononuclear phagocyte system of the mouse. Blood 2003;101:1155-63.

30. Tsukita S, Furuse M, Itoh M. Multifunctional strands in tight junctions. Nat Rev Mol Cell Biol 2001;2:285-93.

31. Lal-Nag M, Morin PJ. The claudins. Genome Biol 2009;10:235.

32. De Benedetto A, Rafaels NM, McGirt LY, Ivanov AI, Georas SN, Cheadle C, et al. Tight junction defects in patients with atopic dermatitis. J Allergy Clin Immunol 2011;127:773-86, e1-7.

33. Chiricozzi A, Nograles KE, Johnson-Huang LM, Fuentes-Duculan J, Cardinale I, Bonifacio KM, et al. IL-17 induces an expanded range of downstream genes in reconstituted human epidermis model. PLoS One 2014;9:e90284.

34. Nograles KE, Suarez-Farinas M, Shemer A, Fuentes-Duculan J, Chiricozzi A, Cardinale I, et al. Atopic dermatitis keratinocytes exhibit normal $\mathrm{T}(\mathrm{H}) 17$ cytokine responses. J Allergy Clin Immunol 2010;125:744-6, e1-746.

35. Fujita H, Nograles KE, Kikuchi T, Gonzalez J, Carucci JA, Krueger JG. Human Langerhans cells induce distinct IL-22-producing CD4 + T cells lacking IL-17 production. Proc Natl Acad Sci U S A 2009;106:21795-800.

36. Forster R, Davalos-Misslitz AC, Rot A. CCR7 and its ligands: balancing immunity and tolerance. Nat Rev Immunol 2008;8:362-71.

37. Guttman-Yassky E, Nograles KE, Krueger JG. Contrasting pathogenesis of atopic dermatitis and psoriasis-part II: immune cell subsets and therapeutic concepts. J Allergy Clin Immunol 2011;127:1420-32.

38. Agrawal R, Woodfolk JA. Skin barrier defects in atopic dermatitis. Curr Allergy Asthma Rep 2014;14:433.

39. Furuse M, Hata M, Furuse K, Yoshida Y, Haratake A, Sugitani Y, et al. Claudin-based tight junctions are crucial for the mammalian epidermal barrier: a lesson from claudin-1-deficient mice. J Cell Biol 2002;156:1099-111. 
40. Yuki T, Komiya A, Kusaka A, Kuze T, Sugiyama Y, Inoue S. Impaired tight junctions obstruct stratum corneum formation by altering polar lipid and profilaggrin processing. J Dermatol Sci 2013;69:148-58.

41. Juric M, Xiao F, Amasheh S, May O, Wahl K, Bantel H, et al. Increased epithelial permeability is the primary cause for bicarbonate loss in inflamed murine colon. Inflamm Bowel Dis 2013;19:904-11.

42. Hou J, Renigunta A, Yang J, Waldegger S. Claudin-4 forms paracellular chloride channel in the kidney and requires claudin-8 for tight junction localization. Proc Natl Acad Sci U S A 2010;107:18010-5.

43. Bronstein JM, Micevych PE, Chen K. Oligodendrocyte-specific protein (OSP) is a major component of CNS myelin. J Neurosci Res 1997;50:713-20.

44. Gow A, Southwood CM, Li JS, Pariali M, Riordan GP, Brodie SE, et al. CNS myelin and sertoli cell tight junction strands are absent in Osp/claudin-11 nul mice. Cell 1999;99:649-59.
45. Kitajiri SI, Furuse M, Morita K, Saishin-Kiuchi Y, Kido H, Ito J, et al. Expression patterns of claudins, tight junction adhesion molecules, in the inner ear. Hear Res 2004; 187:25-34.

46. Kennedy-Crispin M, Billick E, Mitsui H, Gulati N, Fujita H, Gilleaudeau P, et al. Human keratinocytes' response to injury upregulates CCL20 and other genes linking innate and adaptive immunity. J Invest Dermatol 2012;132:105-13.

47. Radoja N, Gazel A, Banno T, Yano S, Blumenberg M. Transcriptional profiling of epidermal differentiation. Physiol Genomics 2006;27:65-78.

48. Liu A. Laser capture microdissection in the tissue biorepository. J Biomol Tech 2010;21:120-5

49. Decarlo K, Emley A, Dadzie OE, Mahalingam M. Laser capture microdissection: methods and applications. Methods Mol Biol 2011;755:1-15.

50. Guttman-Yassky E, Dhingra N, Leung DY. New era of biologic therapeutics in atopic dermatitis. Expert Opin Biol Ther 2013;13:549-61.

\section{Have you seen the new JACI website?}

Enjoy these benefits and more:

- Stay current in your field with Featured Articles of The Week, Articles in Press, and easily view the Most Read and Most Cited articles.

- Sign up for a personalized alerting service with Table of Contents Alerts, Articles in Press Alerts and Saved Search Alerts to be notified when new articles relevant to you are available.

- Create your own Reading List for future reference.

- Link from cited references to abstracts and full text of other participating journals.

- Access additional features such as audio podcasts and the $J A C I$ Journal Club Blog.

Visit www.jacionline.org today to see what else is new online! 